\title{
Global Existence and Asymptotic Behavior of Self-Similar Solutions for the Navier-Stokes-Nernst-Planck-Poisson System in $\mathbb{R}^{3}$
}

\author{
Jihong Zhao, ${ }^{1}$ Chao Deng, ${ }^{2}$ and Shangbin Cui ${ }^{3}$ \\ ${ }^{1}$ Institute of Applied Mathematics, College of Science, Northwest AEF University, Shaanxi, \\ Yangling 712100, China \\ 2 Department of Mathematics, Xuzhou Normal University, Jiangsu, Xuzhou 221009, China \\ ${ }^{3}$ Department of Mathematics, Sun Yat-sen University, Guangdong, Guangzhou 510275, China
}

Correspondence should be addressed to Jihong Zhao, zhaojihong2007@yahoo.com.cn

Received 5 May 2011; Accepted 5 September 2011

Academic Editor: Chérif Amrouche

Copyright (C) 2011 Jihong Zhao et al. This is an open access article distributed under the Creative Commons Attribution License, which permits unrestricted use, distribution, and reproduction in any medium, provided the original work is properly cited.

\begin{abstract}
We study the Navier-Stokes-Nernst-Planck-Poisson system modeling the flow of electrohydrodynamics. For small initial data, the global existence, uniqueness, and asymptotic stability as time goes to infinity of self-similar solutions to the Cauchy problem of this system posed in the whole three dimensional space are proved in the function spaces of pseudomeasure type.
\end{abstract}

\section{Introduction}

In this paper, we consider the Cauchy problem for the (normalized) Navier-Stokes-NernstPlanck-Poisson system which governs the hydrodynamic transport of binary diffusion charge densities as follows (see [1]):

$$
\begin{gathered}
\partial_{t} \mathbf{u}-\Delta \mathbf{u}+(\mathbf{u} \cdot \nabla) \mathbf{u}+\nabla p=\Delta \phi \nabla \phi \text { in } \mathbb{R}^{3} \times(0, \infty), \\
\nabla \cdot \mathbf{u}=0 \quad \text { in } \mathbb{R}^{3} \times(0, \infty), \\
\partial_{t} v+\mathbf{u} \cdot \nabla v=\nabla \cdot(\nabla v-v \nabla \phi) \quad \text { in } \mathbb{R}^{3} \times(0, \infty), \\
\partial_{t} w+\mathbf{u} \cdot \nabla w=\nabla \cdot(\nabla w+w \nabla \phi) \quad \text { in } \mathbb{R}^{3} \times(0, \infty), \\
\Delta \phi=v-w \quad \text { in } \mathbb{R}^{3} \times(0, \infty), \\
\mathbf{u}(x, 0)=\mathbf{u}_{0}(x), \quad v(x, 0)=v_{0}(x), \quad w(x, 0)=w_{0}(x) \text { in } \mathbb{R}^{3} .
\end{gathered}
$$


Equations (1.1) and (1.2) are the momentum conservation and the mass conservation equations of incompressible flow. $\mathbf{u}=\mathbf{u}(x, t)=\left(u_{1}(x, t), u_{2}(x, t), u_{3}(x, t)\right) \in \mathbb{R}^{3}, p=p(x, t) \in \mathbb{R}$ and $\phi=\phi(x, t) \in \mathbb{R}$ denote, respectively, the velocity field, the pressure of the fluid, and the electrostatic potential, and the right-hand side term in (1.1) is the Lorentz force caused by the charged particles. Equations (1.3) and (1.4) model the balance between diffusion and convective transport of charge densities by the flow and the electric fields. $v=v(x, t) \in \mathbb{R}$ and $w=w(x, t) \in \mathbb{R}$ denote the charge densities of the negatively and positively charged species, respectively, hence the sign difference in front of the convective term in either equation. Equation (1.5) is the Poisson equation for the electrostatic potential $\phi$, and the right-hand side is the net charge density. For simplicity, we have chosen the fluid density, viscosity, charge mobility and dielectric constant to be unit.

To start with, let us recall two special cases of (1.1)-(1.6). In the case that the flow is charge free, that is, $v=w=\phi=0$, the system (1.1)-(1.6) reduces into the well-known NavierStokes equations:

$$
\begin{gathered}
\partial_{t} \mathbf{u}-\Delta \mathbf{u}+(\mathbf{u} \cdot \nabla) \mathbf{u}+\nabla P=0 \quad \text { in } \mathbb{R}^{3} \times(0, \infty), \\
\nabla \cdot \mathbf{u}=0 \quad \text { in } \mathbb{R}^{3} \times(0, \infty) \\
\mathbf{u}(x, 0)=\mathbf{u}_{0}(x) \quad \text { in } \mathbb{R}^{3} .
\end{gathered}
$$

After the pioneering work [2], the Navier-Stokes equations (1.7) has drawn great attention of researchers for many years and a huge number of works can be found from the literature, for example, [3-8] and the references therein. If, on the other hand, the velocity field $\mathbf{u}$ is identically vanishing, then (1.1)-(1.6) reduces into the following Nernst-Planck-Poisson equations which was formulated by W. Nernst and M. Planck at the end of the nineteenth century as a basic model for the diffusion of ions in an electrolytes (cf. [9]):

$$
\begin{gathered}
\partial_{t} v=\nabla \cdot(\nabla v-v \nabla \phi) \quad \text { in } \mathbb{R}^{3} \times(0, \infty), \\
\partial_{t} w=\nabla \cdot(\nabla w+w \nabla \phi) \quad \text { in } \mathbb{R}^{3} \times(0, \infty), \\
\Delta \phi=v-w \quad \text { in } \mathbb{R}^{3} \times(0, \infty), \\
v(x, 0)=v_{0}(x), \quad w(x, 0)=w_{0}(x) \quad \text { in } \mathbb{R}^{3} .
\end{gathered}
$$

In some literatures, it is also called the Debye-Hückel system (cf. [10]). It has drawn much attention of analysts during the past twenty years, and some works concerning existence of (large) weak solutions, (small) mild solutions, convergence rate estimates to stationary solutions of time-dependent solutions and other related topics can be found from the literature, cf., for example, [10-16] and the references therein.

In 2002, Jerome [17] proved that the system (1.1)-(1.6) has a unique local smooth solution for smooth initial data where he verified the local existence in Kato's semigroup framework. In [18], by using the energy inequalities and the Schauder fixed point theorem, Schmuck established global existence of weak solutions to the system (1.1)-(1.6) in a bounded domain $\Omega$ with homogeneous Neumann boundary conditions with initial data $\mathbf{u}_{0} \in$ $\left[L^{2}(\Omega)\right]^{n}$ and $v_{0}, w_{0} \in L^{\infty}(\Omega)$ for $n=2,3$. In [19], Ryham studied existence, uniqueness, and regularity of weak solutions of (1.1)-(1.6) in a bounded domain with no-flux boundary conditions for general $L^{2}$ initial data in $n=2$ and for small initial data in $n=3$. The convergence to the stationary solution with a rate is also established in [19]. In our recent work [20], 
by using the $L^{p}-L^{q}$ estimates of the heat semigroup and the classical Hardy-Littlewood-Sobolev inequality, we established local well-posedness of (1.1)-(1.6) in critical and subcritical Lebesgue spaces (i.e., $\mathbf{u}_{0} \in\left[L^{q}\left(\mathbb{R}^{n}\right)\right]^{n}$, and $v_{0}, w_{0} \in L^{p}\left(\mathbb{R}^{n}\right), n \leq q<\infty, n / 2 \leq p<n$ ) and global well-posedness for small initial data in critical Lebesgue spaces (i.e., $\mathbf{u}_{0} \in\left[L^{n}\left(\mathbb{R}^{n}\right)\right]^{n}, v_{0}, w_{0} \in$ $L^{n / 2}\left(\mathbb{R}^{n}\right)$ and $\left\|\mathbf{u}_{0}\right\|_{\left[L^{n}\right]^{n}}+\left\|v_{0}\right\|_{L^{n / 2}}+\left\|w_{0}\right\|_{L^{n / 2}}$ is sufficiently small). For computational simulations of the problem (1.1)-(1.6), see [21-23].

The most important results stated in this paper are theorems on the global existence, uniqueness and asymptotic stability as time goes to infinity of self-similar solutions to the system (1.1)-(1.6) in the functional spaces of pseudomeasure-type. Let us recall that the solution $(\mathbf{u}, p, v, w, \phi)$ of the system (1.1)-(1.6) is called a self-similar solution if it satisfies the following scaling invariant property: $\mathbf{u}(t, x)=\mathbf{u}_{\lambda}(x, t), p(t, x)=p_{\lambda}(x, t), v(t, x)=v_{\lambda}(x, t), w(t, x)=$ $w_{\lambda}(x, t)$ and $\phi(t, x)=\phi_{\lambda}(x, t)$ for all $\lambda>0, x \in \mathbb{R}^{3}$, and $t \geq 0$, where

$$
\begin{gathered}
\mathbf{u}_{\lambda}(x, t)=\lambda \mathbf{u}\left(\lambda x, \lambda^{2} t\right), \quad p_{\lambda}(x, t)=\lambda^{2} p\left(\lambda x, \lambda^{2} t\right), \quad v_{\lambda}(x, t)=\lambda^{2} v\left(\lambda x, \lambda^{2} t\right), \\
w_{\lambda}(x, t)=\lambda^{2} w\left(\lambda x, \lambda^{2} t\right), \quad \phi_{\lambda}(x, t)=\phi\left(\lambda x, \lambda^{2} t\right) .
\end{gathered}
$$

It is clear that if $(\mathbf{u}, p, v, w, \phi)$ is a solution of (1.1)-(1.5) with initial data (1.6), then, for each $\lambda>0,\left(\mathbf{u}_{\curlywedge}, p_{\lambda}, v_{\lambda}, w_{\lambda}, \phi_{\lambda}\right)$ also solves (1.1)-(1.5) with initial data

$$
\mathbf{u}_{0, \lambda}(x)=\lambda \mathbf{u}_{0}(\lambda x), \quad v_{0, \lambda}(x)=\lambda^{2} v_{0}(\lambda x), \quad w_{0, \lambda}(x)=\lambda^{2} w_{0}(\lambda x) .
$$

Apparently such initial data do not belong to any Lebesgue and Sobolev spaces due to their strong singularity at $x=0$ as well as slow decay as $|x| \rightarrow \infty$. In [24], the authors found explicit formulas for a one-parameter family of stationary solutions of the three-dimensional Navier-Stokes equations with zero external force; these solutions are global but not smooth, more precisely, they are singular at the origin with a singularity of the kind $\sim 1 /|x|$ for all time. Note that $1 /|x| \notin L^{3}\left(\mathbb{R}^{3}\right.$ ) but $1 /|x| \in P \mathcal{M}^{2}$ (see (1.16) below for definition of this functional space). Similar phenomenon also appeared for the Nernst-Planck-Poisson equations; see [25]. This is the reason why we consider the system (1.1)-(1.6) in the pseudomeasure-type spaces. By a standard contraction argument, we establish global existence of solution for small initial data. It is worth pointing out that this solution is unique in a ball of the functional spaces in which the existence of solutions is going to be obtained. To overcome this restrictive condition, we establish a stability result in terms of a perturbation of initial data, which allow us to give a complete answer to the uniqueness problem of solution. Moreover, we establish the asymptotic stability of self-similar solutions as time goes to infinity. Here, we refer the reader to see [26-28] and the references cited there for more details related to the NavierStokes equations with measures as initial data.

The self-similar solution is related to an asymptotic behavior, for large time, of global solution to the system (1.1)-(1.6), and we could characterize the self-similar condition in the following way. Here, we disregard the functions $p$ and $\phi$ because when $\mathbf{u}, v$, and $w$ are determined, $p$ and $\phi$ can be easily obtained from (1.2) and (1.5). A vector function $(\mathbf{u}, v, w)$ has the self-similar property to the system (1.1)-(1.6) if and only if there exists a vector function $(\mathbf{U}, V, W)$ such that $\mathbf{u}(x, t)=\mathbf{U}(x / \sqrt{t}) / \sqrt{t}, v(x, t)=V(x / \sqrt{t}) / t$ and $w(x, t)=$ $W(x / \sqrt{t}) / t$ for all $x \in \mathbb{R}^{3}$ and $t>0$. In fact, when $(\mathbf{U}, V, W)$ exists, these last equalities give the definition for $(\mathbf{u}, v, w)$, and it is straightforward to see that it is self-similar. Conversely, when the self-similar solution $(\mathbf{u}, v, w)$ is given, we define $\mathbf{U}(x)=\mathbf{u}(x, 1), V(x)=v(x, 1)$ and 
$W(x)=w(x, 1)$ for all $x \in \mathbb{R}^{3}$. Then, the self-similar condition on $(\mathbf{u}, v, w)$ turns out the expected equality between $(\mathbf{u}, v, w)$ and $(\mathbf{U}, V, W)$ by choosing $\lambda=1 / \sqrt{t}$.

Now, as a standard practice, we can reformulate the problem (1.1)-(1.6) into a system of integral equations. To this end, we first solve (1.5) to get $\phi$ as a functional of $w-v$ :

$$
\phi(x)=(-\Delta)^{-1}(w-v)(x)=\mathcal{F}^{-1}\left(|\xi|^{-2}(\mathcal{F} w(\xi)-\mathcal{F} v(\xi))\right)(x)
$$

in the distributional sense, where $\mathcal{F}$ and $\mathcal{F}^{-1}$ denote the Fourier transform and the inverse Fourier transform, respectively. Next, it is convenient to eliminate the pressure $p$ by applying the Leray projector $\mathbb{P}$ to both sides of (1.1), by (1.11), (1.1) and (1.2) can be transformed into the following equations:

$$
\frac{\partial \mathbf{u}}{\partial t}-\Delta \mathbf{u}+\mathbb{P}(\mathbf{u} \cdot \nabla) \mathbf{u}=\mathbb{P}(v-w) \nabla\left((-\Delta)^{-1}(w-v)\right)
$$

Recalling that $\mathbb{P}$ is given formally by the formula $\mathbb{P}=I+\nabla(-\Delta)^{-1}$ div; that is, $\mathbb{P}$ is the $3 \times 3$ matrix pseudo-differential operator in $\mathbb{R}^{3}$ with the symbol $\mathcal{F}(\mathbb{P})(\xi)=\left(\delta_{j k}-\left(\xi_{j} \xi_{k}\right) /|\xi|^{2}\right)_{j, k=1}^{3}$, where $I$ denotes the unit operator and $\delta_{j k}$ is the Kronecker symbol. It is obvious that all these components are bounded, that is,

$$
\sup _{\xi \in \mathbb{R}^{3} \backslash\{0\}}|F(\mathbb{P})(\xi)|<\infty
$$

Finally, by the well-known Duhamel principle, we see that the problem (1.1)-(1.6) can be further reduced into the following system of integral equations:

$$
\begin{gathered}
\mathbf{u}=e^{t \Delta} \mathbf{u}_{0}+\int_{0}^{t} e^{(t-\tau) \Delta} G_{1}(\mathbf{u}(\tau), v(\tau), w(\tau)) d \tau \\
v=e^{t \Delta} v_{0}+\int_{0}^{t} e^{(t-\tau) \Delta} G_{2}(\mathbf{u}(\tau), v(\tau), w(\tau)) d \tau \\
w=e^{t \Delta} w_{0}+\int_{0}^{t} e^{(t-\tau) \Delta} G_{3}(\mathbf{u}(\tau), v(\tau), w(\tau)) d \tau
\end{gathered}
$$

where $e^{t \Delta}$ is the heat operator which can be regarded as the convolution with the heat kernel $G(x, t)=(4 \pi t)^{-3 / 2} \exp \left(-|x|^{2} / 4 t\right)$, and

$$
\begin{gathered}
G_{1}(\mathbf{u}, v, w)=-\mathbb{P} \nabla \cdot(\mathbf{u} \otimes \mathbf{u})+\mathbb{P}(v-w) \nabla\left((-\Delta)^{-1}(w-v)\right), \\
G_{2}(\mathbf{u}, v, w)=-\nabla \cdot(\mathbf{u} v)-\nabla \cdot\left(v \nabla(-\Delta)^{-1}(w-v)\right), \\
G_{3}(\mathbf{u}, v, w)=-\nabla \cdot(\mathbf{u} w)+\nabla \cdot\left(w \nabla(-\Delta)^{-1}(w-v)\right) .
\end{gathered}
$$

Later on we will work on this system of integral equations instead of (1.1)-(1.6). 
Before giving the explicit meaning of solutions to the system (1.1)-(1.6), we define the functional spaces relevant to the existence of solutions of (1.1)-(1.6). Let us first define

$$
p \mathcal{M}^{a}=\left\{f \in \mathcal{S}^{\prime}\left(\mathbb{R}^{3}\right): \mp f \in L_{\mathrm{loc}}^{1}\left(\mathbb{R}^{3}\right),\|f\|_{p \mathcal{M}^{a}}=\underset{\xi \in \mathbb{R}^{3}}{\operatorname{ess} \sup }|\xi|^{a}|\mp f(\xi)|<\infty\right\}
$$

where $a \geq 0$ is a given parameter. Since $P \mathcal{M}^{a}$ is not separable and the heat semigroup $\left\{e^{t \Delta}\right\}_{t \geq 0}$ is not strongly continuous on this space but only weakly continuous, we will use the notation $\mathcal{C}_{w}$ to denote by the space of functions which are weakly continuous as distributions with respect to $t$. Next, we construct the solution $(\mathbf{u}, v, w)$ of the system (1.1)-(1.6) with the velocity $\mathbf{u}$ in the space

$$
x=\mathcal{C}_{w}\left([0, \infty),\left[D M^{2}\right]^{3}\right)
$$

equipped with the norm $\|\mathbf{u}\|_{\mathcal{X}}=\sup _{t \geq 0}\|\mathbf{u}(t)\|_{\left[p_{\mathcal{M}^{2}}\right]^{3}}$, and the components $v$ and $w$ in the space

$$
y_{a}=\left\{v \in \mathcal{C}_{w}\left([0, \infty), p \mathcal{M}^{1}\right): \sup _{t>0} t^{(a-1) / 2}\|v(t)\|_{p \mathcal{M}^{a}}<\infty\right\}
$$

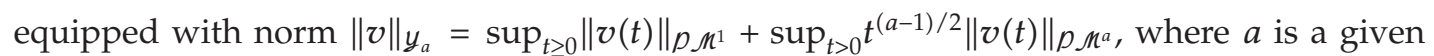
parameter satisfying $1<a<2$ in the whole paper. For simplicity, we denote $\|\cdot\|_{y_{a}, 1}$ and $\|\cdot\|_{y_{a}, 2}$ for each part in the norm of $y_{a}$, and the product of Banach spaces $x \times \mathcal{y} \times \boldsymbol{z}$ will be equipped with the norm $\|(u, v, w)\|_{x \times y \times z}=\max \left\{\|u\|_{x},\|v\|_{y},\|w\|_{z}\right\}$.

Remark 1.1. Let $f \in \mathcal{S}^{\prime}\left(\mathbb{R}^{3}\right) \cap L_{\text {loc }}^{1}\left(\mathbb{R}^{3}\right)$; for a positive parameter $\lambda$, we denote $f_{\lambda}(x)=f(\lambda x)$. It is easy to verify that, for each $\lambda>0$,

$$
\mathcal{F}\left(f_{\curlywedge}\right)(\xi)=\lambda^{-3} \mathcal{F}(f)\left(\lambda^{-1} \xi\right), \quad\|f(\lambda \cdot)\|_{p \mathcal{M}^{a}}=\lambda^{a-3}\|f\|_{p \mathcal{M}^{a}}
$$

Hence, the norms in $x$ and $y_{a}$ are invariant under the scaling (1.9).

Definition 1.2. The solution of (1.1)-(1.6) is a vector function $(\mathbf{u}, v, w)$ with components satisfying, for some $0<T \leq \infty$,

$$
\mathbf{u} \in \mathcal{C}_{w}\left([0, T),\left[p \mathcal{M}^{2}\right]^{3}\right), \quad v, w \in \mathcal{C}_{w}\left([0, T), P \mathcal{M}^{1}\right)
$$

and the following equalities hold for all $0 \leq t<T$ :

$$
\begin{aligned}
\mathcal{F}(\mathbf{u})(\xi, t)= & e^{-t|\xi|^{2}} \mathcal{F}\left(\mathbf{u}_{0}\right)(\xi)-\int_{0}^{t} e^{-(t-\tau)|\xi|^{2}} \mathcal{F}(\mathbb{P})(\xi) i \xi \cdot \mathcal{F}(\mathbf{u} \otimes \mathbf{u})(\xi, \tau) d \tau \\
& +\int_{0}^{t} e^{-(t-\tau)|\xi|^{2}} \mathcal{F}(\mathbb{P})(\xi) \mathcal{F}\left((v-w) \nabla\left((-\Delta)^{-1}(w-v)\right)\right)(\xi, \tau) d \tau,
\end{aligned}
$$




$$
\begin{aligned}
\mathcal{F}(v)(\xi, t)= & e^{-t|\xi|^{2}} \mathcal{F}\left(v_{0}\right)(\xi)-\int_{0}^{t} e^{-(t-\tau)|\xi|^{2}} i \xi \cdot \mathcal{F}(\mathbf{u} v)(\xi, \tau) d \tau \\
& -\int_{0}^{t} e^{-(t-\tau)|\xi|^{2}} i \xi \cdot \mathcal{F}\left(v \nabla\left((-\Delta)^{-1}(w-v)\right)\right)(\xi, \tau) d \tau \\
\mathcal{F}(w)(\xi, t)= & e^{-t|\xi|^{2}} \mathcal{F}\left(w_{0}\right)(\xi)-\int_{0}^{t} e^{-(t-\tau)|\xi|^{2}} i \xi \cdot \mathcal{F}(\mathbf{u} w)(\xi, \tau) d \tau \\
& +\int_{0}^{t} e^{-(t-\tau)|\xi|^{2}} i \xi \cdot \mathcal{F}\left(w \nabla\left((-\Delta)^{-1}(w-v)\right)\right)(\xi, \tau) d \tau .
\end{aligned}
$$

Now, we state the main results of this paper as follows.

Theorem 1.3. Let $\mathbf{u}_{0} \in\left[P \mathcal{M}^{2}\right]^{3}, \nabla \cdot \mathbf{u}_{0}=0$, and $v_{0}, w_{0} \in P \mathcal{M}^{1}$. There exists a constant $\varepsilon$ such that if $\left\|\left(\mathbf{u}_{0}, v_{0}, w_{0}\right)\right\|_{\left[p \mathcal{M}^{2}\right]^{3} \times\left[p \mathcal{M}^{1}\right]^{2}} \leq \varepsilon$, then the system $(1.1)-(1.6)$ has a global solution $(\mathbf{u}, \boldsymbol{v}, \boldsymbol{w})$ in the space $\mathcal{X} \times\left[y_{a}\right]^{2}$, and this is the unique solution under the condition

$$
\|(\mathbf{u}, v, w)\|_{\mathcal{X} \times\left[y_{a}\right]^{2}} \leq 2 \eta \varepsilon,
$$

where $0<\varepsilon<1 /\left(4 \eta^{2}\right)$ and the constant $\eta$ is defined by (2.23). Moreover, the solution depends continuously on initial data in the following sense: if $\left\|\left(\widetilde{\mathbf{u}}_{0}, \widetilde{v}_{0}, \widetilde{w}_{0}\right)\right\|_{\left[p \Re^{2}\right]^{3} \times\left[p \mathcal{M}^{1}\right]^{2}} \leq \varepsilon$, then one denotes by $(\widetilde{\mathbf{u}}, \widetilde{v}, \widetilde{w})$ the unique solution of $(1.1)-(1.6)$ with initial data $\left(\widetilde{\mathbf{u}}_{0}, \widetilde{v}_{0}, \widetilde{w}_{0}\right)$, and $\|(\widetilde{\mathbf{u}}, \widetilde{v}, \widetilde{w})\|_{\mathcal{X}\left[y_{a}\right]^{2}} \leq$ $2 \eta \varepsilon$, then, one has

$$
\|(\mathbf{u}-\tilde{\mathbf{u}}, v-\tilde{v}, w-\tilde{w})\|_{\mathcal{X} \times\left[y_{a}\right]^{2}} \leq C(\varepsilon, \eta)\left\|\left(\mathbf{u}_{0}-\tilde{\mathbf{u}}_{0}, v_{0}-\widetilde{v}_{0}, w_{0}-\tilde{w}_{0}\right)\right\|_{\left[p \mathcal{M}^{2}\right]^{3} \times\left[p \mathcal{M}^{1}\right]^{2}},
$$

where $C(\varepsilon, \eta)=\eta /\left(1-4 \varepsilon \eta^{2}\right)$.

Remark 1.4. In Theorem 1.3, we obtained only a partial answer to the uniqueness problem of solution; that is, under the restrictive condition (1.22), the solution of (1.1)-(1.6) is unique. For a complete answer to this problem, see Corollary 1.8 below.

Based on the uniqueness of solution in Theorem 1.3, by a standard way, we can deduce the existence of self-similar solution to the system (1.1)-(1.6).

Corollary 1.5. Assume that $\mathbf{u}_{0}, v_{0}$, and $w_{0}$ satisfy the assumptions of Theorem 1.3. Assume that, moreover,

$$
\mathbf{u}_{0}(x)=\lambda \mathbf{u}_{0}(\lambda x), \quad v_{0}(x)=\lambda^{2} v_{0}(\lambda x), \quad w_{0}(x)=\lambda^{2} w_{0}(\lambda x)
$$

Then, the solution $(\mathbf{u}, v, w)$ constructed in Theorem 1.3 is a self-similar solution.

In order to give a complete answer to the uniqueness problem of solutions to the system (1.1)-(1.6), we will establish the following stability result.

Theorem 1.6. Let $\left(\mathbf{u}_{0}, v_{0}, w_{0}\right)$ and $\left(\widetilde{\mathbf{u}}_{0}, \widetilde{v}_{0}, \widetilde{w}_{0}\right)$ belong to $\left[D \mathcal{M}^{2}\right]^{3} \times\left[D \mathcal{M}^{1}\right]^{2}$ such that $\|\left(\mathbf{u}_{0}-\widetilde{\mathbf{u}}_{0}, v_{0}-\right.$ $\left.\widetilde{v}_{0}, w_{0}-\widetilde{w}_{0}\right) \|_{\left[p M^{2}\right]^{3} \times\left[p \mathcal{M}^{1}\right]^{2}}<1 /\left(8 \eta^{2}\right)$, where $\eta$ is a constant defined by $(2.23)$, and let $(\mathbf{u}, v, w)$ and 
$(\widetilde{\mathbf{u}}, \widetilde{v}, \widetilde{w})$ be two solutions of $(1.1)-(1.6)$ with initial conditions $\left(\mathbf{u}_{0}, \boldsymbol{v}_{0}, w_{0}\right)$ and $\left(\widetilde{\mathbf{u}}_{0}, \widetilde{v}_{0}, \widetilde{w}_{0}\right)$, respectively. Then, one has

$$
\|(\mathbf{u}-\tilde{\mathbf{u}}, v-\tilde{v}, w-\tilde{w})\|_{\mathcal{X} \times\left[y_{a}\right]^{2}} \leq 2 \eta\left\|\left(\mathbf{u}_{0}-\tilde{\mathbf{u}}_{0}, v_{0}-\tilde{v}_{0}, w_{0}-\tilde{w}_{0}\right)\right\|_{\left[D \Re^{2}\right]^{3} \times\left[p \mathcal{M}^{1}\right]^{2}}
$$

Remark 1.7. Theorem 1.6 implies that we can measure the difference of two solutions in terms of the difference of their initial data provided that the difference between these initial data is small enough.

The direct consequence of Theorem 1.6 is the following corollary.

Corollary 1.8. Assume that $\mathbf{u}_{0} \in\left[P \mathcal{M}^{2}\right]^{3}, \nabla \cdot \mathbf{u}_{0}=0$ in the distributional sense, and $v_{0}, w_{0} \in P \mathcal{M}^{1}$. Then, there exists at most one solution of (1.1)-(1.6) with initial data $\left(\mathbf{u}_{0}, v_{0}, w_{0}\right)$.

Finally, we study the asymptotic stability of solutions in the sense proposed in [13] and developed in [27].

Theorem 1.9. Let $\varepsilon$ be a sufficiently small number such that $\varepsilon<\min \left\{1 /\left(4 \eta^{2}\right), 1 / \tilde{\eta}\right\}$, where $\eta$ and $\tilde{\eta}$ are defined by $(2.23)$ and $(4.19)$, respectively. Assume that $\left(\mathbf{u}_{0}, v_{0}, w_{0}\right)$ and $\left(\widetilde{\mathbf{u}}_{0}, \widetilde{v}_{0}, \widetilde{w}_{0}\right)$ satisfy the assumptions of Theorem 1.3, and let $(\mathbf{u}, v, w)$ and $(\tilde{\mathbf{u}}, \widetilde{v}, \widetilde{w})$ be two global solutions of (1.1)-(1.6) with initial conditions $\left(\mathbf{u}_{0}, v_{0}, w_{0}\right)$ and $\left(\widetilde{\mathbf{u}}_{0}, \widetilde{v}_{0}, \widetilde{w}_{0}\right)$, respectively. Then, the following two conditions are equivalent:

$$
\begin{aligned}
& \lim _{t \rightarrow \infty}\left(\left\|e^{t \Delta}\left(\mathbf{u}_{0}-\tilde{\mathbf{u}}_{0}, v_{0}-\widetilde{v}_{0}, w_{0}-\widetilde{w}_{0}\right)\right\|_{\left[p \mathcal{M}^{2}\right]^{3} \times\left[p \mathcal{M}^{1}\right]^{2}}+t^{(a-1) / 2}\right. \\
& \left.\times\left\|e^{t \Delta}\left(v_{0}-\widetilde{v}_{0}, w_{0}-\widetilde{w}_{0}\right)\right\|_{\left[p \Re^{a}\right]^{2}}\right)=0, \\
& \lim _{t \rightarrow \infty}\left(\|(\mathbf{u}-\tilde{\mathbf{u}}, v-\tilde{v}, w-\tilde{w})\|_{\left[p M^{2}\right]^{3} \times\left[p M^{1}\right]^{2}}+t^{(a-1) / 2}\|(v-\tilde{v}, w-\tilde{w})\|_{\left[p M^{a}\right]^{2}}\right)=0 .
\end{aligned}
$$

Remark 1.10. As an interesting application of Theorem 1.9, we get the asymptotic stability of self-similar solutions to the system (1.1)-(1.6), namely, under the assumptions of Theorem 1.9, besides, assume that $\left(\mathbf{u}_{0}, v_{0}, w_{0}\right)$ satisfies (1.24). Then, we know that the mild solution $(\widetilde{\mathbf{u}}, \widetilde{v}, \widetilde{w})$ tends to the self-similar solution $(\mathbf{u}, v, w)$ as time goes to infinity as long as $\left(\widetilde{\mathbf{u}}_{0}, \widetilde{v}_{0}, \widetilde{w}_{0}\right)$ satisfies the condition (1.26).

\section{Notations}

Let $A$ and $B$ be two real numbers; we denote $A \lesssim B$ if there is a universal constant $C$, which does not depend on varying parameters of the problem, such that $A \leq C B$. We denote $A \sim B$ if $A \lesssim B$ and $B \lesssim A$. In the rest part of this paper, we will use "sup" instead of "ess sup" for convenience.

\section{Structure of the Paper}

In Section 2, we prove Theorem 1.3 and Corollary 1.5. The purpose of Section 3 is to prove Theorem 1.6. In the last section, we present the proof of Theorem 1.9. 


\section{Global-in-Time Solutions}

In this section, we give the proofs of Theorem 1.3 and Corollary 1.5. Thus, throughout this section, we assume that $\mathbf{u}_{0} \in\left[P \mathcal{M}^{2}\right]^{3}, \nabla \cdot \mathbf{u}_{0}=0$, and $v_{0}, w_{0} \in P \mathcal{M}^{1}$.

\subsection{The Proof of Theorem 1.3}

Let $a$ be a fixed number such that $1<a<2$, and let $\mathfrak{X}=\mathcal{X} \times\left[y_{a}\right]^{2}$. Given $(\mathbf{u}, v, w) \in \mathfrak{X}$, we define $\mathfrak{F}(\mathbf{u}, v, w)=(\overline{\mathbf{u}}, \bar{v}, \bar{w})$, where

$$
\begin{aligned}
& \overline{\mathbf{u}}=e^{t \Delta} \mathbf{u}_{0}+\int_{0}^{t} e^{(t-\tau) \Delta} G_{1}(\mathbf{u}(\tau), v(\tau), w(\tau)) d \tau \\
& \bar{v}=e^{t \Delta} v_{0}+\int_{0}^{t} e^{(t-\tau) \Delta} G_{2}(\mathbf{u}(\tau), v(\tau), w(\tau)) d \tau \\
& \bar{w}=e^{t \Delta} w_{0}+\int_{0}^{t} e^{(t-\tau) \Delta} G_{3}(\mathbf{u}(\tau), v(\tau), w(\tau)) d \tau .
\end{aligned}
$$

We fulfil the proof of Theorem 1.3 through the following two lemmas.

Lemma 2.1. The map $\mathfrak{F}$ is well defined and maps $\mathfrak{X}$ into itself.

Proof. Note that although some parts of the proof were given in [27], we would rather give it for completeness. We first prove that $\overline{\mathbf{u}}$ is well defined and $\overline{\mathbf{u}} \in \mathcal{X}$. From (2.1), we can denote $\overline{\mathbf{u}}=\overline{\mathbf{u}}_{1}+\overline{\mathbf{u}}_{2}+\overline{\mathbf{u}}_{3}$, where

$$
\begin{gathered}
\overline{\mathbf{u}}_{1}(t)=e^{t \Delta} \mathbf{u}_{0}, \\
\overline{\mathbf{u}}_{2}(t)=\int_{0}^{t} e^{(t-\tau) \Delta} \mathbb{P}[-\nabla \cdot(\mathbf{u} \otimes \mathbf{u})](\tau) d \tau, \\
\overline{\mathbf{u}}_{3}(t)=\int_{0}^{t} e^{(t-\tau) \Delta} \mathbb{P}\left[(v-w) \nabla\left((-\Delta)^{-1}(w-v)\right)\right](\tau) d \tau .
\end{gathered}
$$

For $\overline{\mathbf{u}}_{1}$, since $\mathbf{u}_{0} \in\left[D \mathcal{M}^{2}\right]^{3}$, it is easy to see that

$$
\left\|e^{t \Delta} \mathbf{u}_{0}\right\|_{\chi}=\sup _{t \geq 0} \sup _{\xi \in \mathbb{R}^{3}}|\xi|^{2}\left|e^{-t|\xi|^{2}} \boldsymbol{F}\left(\mathbf{u}_{0}\right)(\xi)\right| \leq\left\|\mathbf{u}_{0}\right\|_{\left[p \mathcal{M}^{2}\right]^{3}} .
$$

Thus, $e^{t \Delta} \mathbf{u}_{0} \in L^{\infty}\left([0, \infty),\left[P \mathcal{M}^{2}\right]^{3}\right)$. To prove the weak continuity of $\overline{\mathbf{u}}_{1}$ with respect to $t$, due to the properties of heat semigroup $e^{t \Delta}$, it suffices to prove this for $t=0$. For every $\varphi \in\left[\mathcal{S}\left(\mathbb{R}^{3}\right)\right]^{3}$, by applying the Plancherel formula, we obtain

$$
\begin{aligned}
\left|\left\langle e^{t \Delta} \mathbf{u}_{0}-\mathbf{u}_{0}, \varphi\right\rangle\right| & =\left|\int_{\mathbb{R}^{3}}\left(e^{-t|\xi|^{2}}-1\right) \mathcal{F}\left(\mathbf{u}_{0}\right)(\xi) \mathcal{F}(\varphi)(\xi) d \xi\right| \\
& \leq t \sup _{\xi \in \mathbb{R}^{3}}\left|\frac{\left(e^{-t|\xi|^{2}}-1\right)}{\left(t|\xi|^{2}\right)}\right|\left\|\mathbf{u}_{0}\right\|_{\left[p \mathscr{M}^{2}\right]^{3}}\|\mathcal{F}(\varphi)\|_{\left[L^{1}\left(\mathbb{R}^{3}\right)\right]^{3}} \longrightarrow 0 \quad \text { as } t \longrightarrow 0 .
\end{aligned}
$$


This implies that

$$
\overline{\mathbf{u}}_{1} \in \mathcal{X}, \quad\left\|\overline{\mathbf{u}}_{1}\right\|_{\mathcal{X}} \leq\left\|\mathbf{u}_{0}\right\|_{\left[P \mathcal{M}^{2}\right]^{3}} .
$$

For $\overline{\mathbf{u}}_{2}$, using (1.13) and the properties of the Fourier transform, we get

$$
\begin{aligned}
\left\|\overline{\mathbf{u}}_{2}\right\|_{\left[D \mathscr{M}^{2}\right]^{3}} & \sup _{\xi \in \mathbb{R}^{3}}|\xi|^{2}\left|\int_{0}^{t} e^{-(t-\tau)|\xi|^{2}} \boldsymbol{\Psi}(\mathbb{P})(\xi) i \xi \cdot \boldsymbol{F}(\mathbf{u} \otimes \mathbf{u})(\xi, \tau) d \tau\right| \\
& \lesssim \sup _{\xi \in \mathbb{R}^{3}} \int_{0}^{t}|\xi|^{3} e^{-(t-\tau)|\xi|^{2}}\left(|\xi|^{-2} *|\xi|^{-2}\right) d \tau\|\mathbf{u}\|_{X}^{2} \\
& \lesssim \sup _{\xi \in \mathbb{R}^{3}} \int_{0}^{t}|\xi|^{2} e^{-(t-\tau)|\xi|^{2}} d \tau\|\mathbf{u}\|_{X}^{2} \\
& \lesssim\|\mathbf{u}\|_{X}^{2} .
\end{aligned}
$$

Here, we have used the fact that $|\xi|^{-2} *|\xi|^{-2} \sim|\xi|^{-1}$ (see [29, Chapter 5, Section 1, (8)]). For the weak continuity of $\overline{\mathbf{u}}_{2}$ with respect to $t$, we can prove it by a standard argument (cf. [30]). Hence,

$$
\overline{\mathbf{u}}_{2} \in \mathcal{X}, \quad\left\|\overline{\mathbf{u}}_{2}\right\|_{\mathcal{X}} \lesssim\|\mathbf{u}\|_{\mathcal{X}}^{2} .
$$

For $\overline{\mathbf{u}}_{3}$, using similar calculations,

$$
\begin{aligned}
\left\|\overline{\mathbf{u}}_{3}\right\|_{\left[D \mathcal{M}^{2}\right]^{3}} & =\sup _{\xi \in \mathbb{R}^{3}}|\xi|^{2}\left|\int_{0}^{t} e^{-(t-\tau)|\xi|^{2}} \mathcal{F}(\mathbb{P})(\xi) \mathcal{F}\left((v-w) \nabla\left((-\Delta)^{-1}(w-v)\right)\right)(\xi, \tau) d \tau\right| \\
& \lesssim \sup _{\xi \in \mathbb{R}^{3}} \int_{0}^{t}|\xi|^{2} e^{-(t-\tau)|\xi|^{2}}\left(|\xi|^{-a} *|\xi|^{-a-1}\right) \tau^{-(a-1)} d \tau\|(v, w)\|_{y_{a, 2}}^{2} \\
& \lesssim \sup _{\xi \in \mathbb{R}^{3}} \int_{0}^{t}|\xi|^{4-2 a} e^{-(t-\tau)|\xi|^{2}} \tau^{-(a-1)} d \tau\|(v, w)\|_{y_{a}, 2}^{2} \\
& \lesssim \sup _{\xi \in \mathbb{R}^{3}} \int_{0}^{t}\left((t-\tau)|\xi|^{2}\right)^{2-a} e^{-(t-\tau)|\xi|^{2}}(t-\tau)^{-(2-a)} \tau^{-(a-1)} d \tau\|(v, w)\|_{y_{a}, 2}^{2} \\
& \lesssim\|(v, w)\|_{y_{a, 2} .}^{2}
\end{aligned}
$$

Here, we have used the fact $|\xi|^{-a} *|\xi|^{-a-1} \sim|\xi|^{2-2 a}$ and the assumption $1<a<2$ to ensure that the integral $\int_{0}^{t}(t-\tau)^{-(2-a)} \tau^{-(a-1)} d \tau$ is finite and independent of $t$. It remains to show the weak continuity of $\overline{\mathbf{u}}_{3}$, but this is a standard argument as we mentioned before. Hence,

$$
\overline{\mathbf{u}}_{3} \in \mathcal{X}, \quad\left\|\overline{\mathbf{u}}_{3}\right\|_{\mathcal{X}} \lesssim\|(v, w)\|_{y_{a}, 2}^{2}
$$


Combining the above estimates (2.7)-(2.11), we see that

$$
\overline{\mathbf{u}} \in \mathcal{X}, \quad\|\overline{\mathbf{u}}\|_{\mathcal{X}} \lesssim\left\|\mathbf{u}_{0}\right\|_{\left[p \mathcal{M}^{2}\right]^{3}}+\|\mathbf{u}\|_{X}^{2}+\|(v, w)\|_{y_{a}, 2}^{2} .
$$

Next, we prove that $\bar{v}$ is well defined and $\bar{v} \in \mathcal{y}_{a}$. Note that, from (2.2), we can denote $\bar{v}=\bar{v}_{1}+\bar{v}_{2}+\bar{v}_{3}$, where

$$
\begin{gathered}
\bar{v}_{1}(t)=e^{t \Delta} v_{0} \\
\bar{v}_{2}(t)=\int_{0}^{t} e^{(t-\tau) \Delta}[-\nabla \cdot(\mathbf{u} v)](\tau) d \tau \\
\bar{v}_{3}(t)=\int_{0}^{t} e^{(t-\tau) \Delta}\left[-\nabla \cdot\left(v \nabla\left((-\Delta)^{-1}(w-v)\right)\right)\right](\tau) d \tau .
\end{gathered}
$$

Since $v_{0} \in P \mathcal{M}^{1}$, as in the proof of $\overline{\mathbf{u}}_{1}$, it can be easily seen that

$$
\bar{v}_{1} \in \mathcal{Y}_{a}, \quad\left\|\bar{v}_{1}\right\|_{y_{a}} \lesssim\left\|v_{0}\right\|_{p \Re^{1}}
$$

Indeed, it suffices to estimate the second term in the norm of $y_{a}$ as follows:

$$
\begin{aligned}
\sup _{t>0} t^{(a-1) / 2}\left\|\bar{v}_{1}\right\|_{p \mathcal{M}^{a}} & =\sup _{t>0} \sup _{\xi \in \mathbb{R}^{3}} t^{(a-1) / 2}|\xi|^{a} e^{-t|\xi|^{2}}\left|\mathcal{F}\left(v_{0}\right)(\xi)\right| \\
& \leq \sup _{t>0} \sup _{\xi \in \mathbb{R}^{3}}\left(t|\xi|^{2}\right)^{(a-1) / 2} e^{-t|\xi|^{2}}|\xi|\left|\mathcal{F}\left(v_{0}\right)(\xi)\right| \lesssim\left\|v_{0}\right\|_{p \mathcal{M}^{1}} .
\end{aligned}
$$

For $\bar{v}_{2}$, we can do the same calculations to deal with the first term in the norm of $y_{a}$, and one obtains that

$$
\left\|\bar{v}_{2}\right\|_{y_{a, 1}} \lesssim\|\mathbf{u}\|_{\mathcal{X}}\|v\|_{y_{a, 2}}
$$

To deal with the second term in the norm of $y_{a}$, we need to calculate more. Note first the following two elementary inequalities:

$$
\begin{aligned}
& |\xi|^{2-a} \int_{0}^{t / 2} e^{-(t-\tau)|\xi|^{2}} \tau^{-(a-1) / 2} d \tau \leq|\xi|^{2-a} e^{-\left(t|\xi|^{2}\right) / 2} \int_{0}^{t / 2} \tau^{-(a-1) / 2} d \tau \lesssim|\xi|^{-a} t^{-(a-1) / 2}, \\
& |\xi|^{2-a} \int_{t / 2}^{t} e^{-(t-\tau)|\xi|^{2}} \tau^{-(a-1) / 2} d \tau \lesssim|\xi|^{2-a} t^{-(a-1) / 2} \int_{t / 2}^{t} e^{-(t-\tau)|\xi|^{2}} d \tau \lesssim|\xi|^{-a} t^{-(a-1) / 2} .
\end{aligned}
$$

Hence, taking the Fourier transform to $\bar{v}_{2}$, we get

$$
\begin{aligned}
& \left|\mathcal{F}\left(\bar{v}_{2}\right)\right| \lesssim \int_{0}^{t}|\xi| e^{-(t-\tau)|\xi|^{2}}\left(|\xi|^{-2} *|\xi|^{-a}\right) \tau^{-(a-1) / 2} d \tau\|\mathbf{u}\|_{\chi}\|v\|_{y_{a, 2}}
\end{aligned}
$$

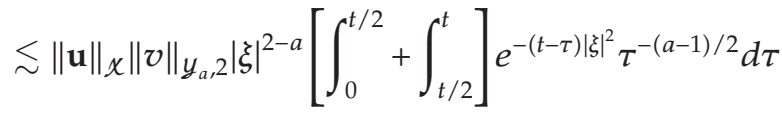

$$
\begin{aligned}
& \lesssim|\xi|^{-a} t^{-(a-1) / 2}\|\mathbf{u}\|_{\chi}\|v\|_{y_{a, 2}} \text {. }
\end{aligned}
$$


This implies that $\left\|\bar{v}_{2}\right\|_{y_{a}, 2} \lesssim\|\mathbf{u}\| x\|v\|_{y_{a}, 2}$. By a standard argument, we can prove that $\bar{v}_{2}$ is weakly continuous with respect to $t$. Hence,

$$
\bar{v}_{2} \in y_{a}, \quad\left\|\bar{v}_{2}\right\|_{y_{a}} \lesssim\|\mathbf{u}\|_{\mathcal{X}}\|v\|_{y_{a}, 2} .
$$

For $\bar{v}_{3}$, we can do the same calculations as for $\bar{v}_{2}$ and obtain

$$
\bar{v}_{3} \in y_{a}, \quad\left\|\bar{v}_{3}\right\|_{y_{a}} \lesssim\|(v, w)\|_{y_{a}, 2}^{2}
$$

Concluding the above estimates (2.14)-(2.20), we have already proved that

$$
\bar{v} \in y_{a}, \quad\|\bar{v}\|_{y_{a}} \lesssim\left\|v_{0}\right\|_{p_{\mathcal{M}^{1}}}+\left(\|\mathbf{u}\|_{\mathcal{X}}+\|(v, w)\|_{y_{a}, 2}\right)\|(v, w)\|_{y_{a}, 2}
$$

Similarly, for $\bar{w}$, we can prove that

$$
\bar{w} \in \mathcal{Y}_{a}, \quad\|\bar{w}\|_{y_{a}} \lesssim\left\|w_{0}\right\|_{p_{\mathcal{M}^{1}}}+\left(\|\mathbf{u}\|_{\mathcal{X}}+\|(v, w)\|_{y_{a, 2}}\right)\|(v, w)\|_{y_{a}, 2} .
$$

The proof of Lemma 2.1 is complete by (2.12), (2.21), and (2.22).

From Lemma 2.1, there exists a constant $\eta>0$ such that, for any $(\mathbf{u}, v, w) \in \mathfrak{X}$ and $(\overline{\mathbf{u}}, \bar{v}, \bar{w})=\mathfrak{F}(\mathbf{u}, v, w)$, one has

$$
\|(\overline{\mathbf{u}}, \bar{v}, \bar{w})\|_{\mathfrak{X}} \leq \eta\left(\left\|\left(\mathbf{u}_{0}, v_{0}, w_{0}\right)\right\|_{\left[P M^{2}\right]^{3} \times\left[P \mathcal{M}^{1}\right]^{2}}+\|(\mathbf{u}, v, w)\|_{\mathfrak{X}}^{2}\right) .
$$

Let $\varepsilon>0$ be sufficiently small so that $4 \eta^{2} \varepsilon<1$. If $\left\|\left(\mathbf{u}_{0}, v_{0}, w_{0}\right)\right\|_{\left[D M^{2}\right]^{3} \times\left[D M^{1}\right]^{2}} \leq \varepsilon$, then from (2.23) one has

$$
\|(\overline{\mathbf{u}}, \bar{v}, \bar{w})\|_{\mathfrak{X}} \leq \eta \varepsilon+\eta\|(\mathbf{u}, v, w)\|_{\mathfrak{X}}^{2} .
$$

Now, let $\mathfrak{B}$ be a closed ball in $\mathfrak{X}$ with radius $2 \eta \mathcal{E}$, that is,

$$
\mathfrak{B}=\left\{(\mathbf{u}, v, w) \in \mathfrak{X}:\|(\mathbf{u}, v, w)\|_{\mathfrak{X}} \leq 2 \eta \varepsilon\right\} .
$$

For any $(\mathbf{u}, v, w) \in \mathfrak{B}$, from $(2.24)$, we see that

$$
\|(\overline{\mathbf{u}}, \bar{v}, \bar{w})\|_{\mathfrak{X}} \leq \eta \varepsilon+\eta(2 \eta \varepsilon)^{2}=\left(1+4 \eta^{2} \varepsilon\right) \eta \varepsilon \leq 2 \eta \varepsilon
$$

This implies that $\mathfrak{F}$ maps $\mathfrak{B}$ into itself.

Lemma 2.2. Let $\varepsilon$ be as before $\left(\varepsilon<1 / 4 \eta^{2}\right)$. If $\left\|\left(\mathbf{u}_{0}, v_{0}, w_{0}\right)\right\|_{\left[p \mathcal{M}^{2}\right]^{3} \times\left[p \mathcal{M}^{1}\right]^{2}} \leq \varepsilon$, then $\mathfrak{F}$ is a contraction mapping. 
Proof. Let $\left(\mathbf{u}_{1}, v_{1}, w_{1}\right),\left(\mathbf{u}_{2}, v_{2}, w_{2}\right) \in \mathfrak{B}$, and let $\left(\overline{\mathbf{u}}_{j}, \overline{\boldsymbol{v}}_{j}, \bar{w}_{j}\right)=\mathfrak{F}\left(\mathbf{u}_{j}, \boldsymbol{v}_{j}, w_{j}\right), j=1,2$. Then by a similar argument as in the proof of Lemma 2.1, we obtain the following estimate:

$$
\begin{aligned}
\left\|\left(\overline{\mathbf{u}}_{1}-\overline{\mathbf{u}}_{2}, \bar{v}_{1}-\bar{v}_{2}, \bar{w}_{1}-\bar{w}_{2}\right)\right\|_{\mathfrak{X}} \leq & \eta\left(\left\|\left(\mathbf{u}_{1}, v_{1}, w_{1}\right)\right\|_{\mathfrak{X}}+\left\|\left(\mathbf{u}_{2}, v_{2}, w_{2}\right)\right\|_{\mathfrak{X}}\right) \\
& \times\left\|\left(\mathbf{u}_{1}-\mathbf{u}_{2}, v_{1}-v_{2}, w_{1}-w_{2}\right)\right\|_{\mathfrak{X}} \\
\leq & 4 \eta^{2} \varepsilon\left\|\left(\mathbf{u}_{1}-\mathbf{u}_{2}, v_{1}-v_{2}, w_{1}-w_{2}\right)\right\|_{\mathfrak{X}}
\end{aligned}
$$

Since $4 \eta^{2} \varepsilon<1$, we see that $\mathfrak{F}$ is a contraction mapping. By using Lemmas 2.1 and 2.2 and the Banach fixed point theorem, we know there exists a global solution $(\mathbf{u}, v, w)$ of $(1.1)-(1.6)$ in the space $\mathcal{X} \times\left[y_{a}\right]^{2}$, and this is the unique solution satisfying the condition $\|(\mathbf{u}, v, w)\|_{\chi_{x}\left[y_{a}\right]^{2}} \leq$ $2 \eta \varepsilon$. It remains to show that the solution depends continuously on initial data. Let $(\mathbf{u}, v, w)$ and $(\widetilde{\mathbf{u}}, \widetilde{v}, \widetilde{w})$ be two solutions of (1.1)-(1.6) corresponding to initial conditions $\left(\mathbf{u}_{0}, v_{0}, w_{0}\right)$ and $\left(\widetilde{\mathbf{u}}_{0}, \widetilde{v}_{0}, \widetilde{w}_{0}\right)$, respectively, and

$$
\left\|\left(\mathbf{u}_{0}, v_{0}, w_{0}\right)\right\|_{\left[p \mathcal{M}^{2}\right]^{3} \times\left[p \mathcal{M}^{1}\right]^{2}} \leq \varepsilon, \quad\left\|\left(\tilde{\mathbf{u}}_{0}, \widetilde{v}_{0}, \widetilde{w}_{0}\right)\right\|_{\left[p \mathcal{M}^{2}\right]^{3} \times\left[p \mathcal{M}^{1}\right]^{2}} \leq \varepsilon
$$

Then, proceeding as in (2.27), we get

$$
\begin{aligned}
& \|(\mathbf{u}-\tilde{\mathbf{u}}, v-\tilde{v}, w-\tilde{w})\|_{\chi \times\left[y_{a}\right]^{2}} \leq \eta\left\|\left(\mathbf{u}_{0}-\tilde{\mathbf{u}}_{0}, v_{0}-\widetilde{v}_{0}, w_{0}-\tilde{w}_{0}\right)\right\|_{\left[D M^{2}\right]^{3} \times\left[D M^{1}\right]^{2}} \\
& +4 \eta^{2} \varepsilon\|(\mathbf{u}-\widetilde{\mathbf{u}}, v-\widetilde{v}, w-\widetilde{w})\|_{\mathfrak{X}} .
\end{aligned}
$$

Since $4 \eta^{2} \varepsilon<1,(2.29)$ yields that

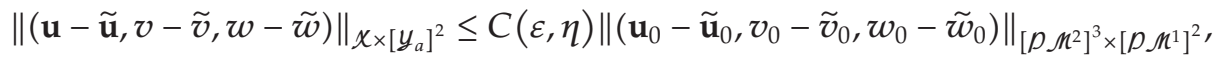

where $C(\varepsilon, \eta)=\eta /\left(1-4 \varepsilon \eta^{2}\right)$. This proves Theorem 1.3.

\subsection{The Proof of Corollary 1.5}

Proof. On the one hand, from Theorem 1.3, we know, the system (1.1)-(1.6) admits a unique global solution $(\mathbf{u}, v, w)$ with initial data $\left(\mathbf{u}_{0}, v_{0}, w_{0}\right)$. Moreover, $\|(\mathbf{u}, v, w)\|_{\mathcal{X} \times\left[y_{a}\right]^{2}} \leq 2 \eta \varepsilon$. On the other hand, since $\mathbf{u}_{0}, v_{0}$, and $w_{0}$ satisfy the condition (1.24), by the scaling invariance of (1.1)-(1.6), for each $\lambda>0$, the function $\left(\mathbf{u}_{\curlywedge}, v_{\lambda}, w_{\curlywedge}\right)$ (see (1.9)) is also a solution with the same initial data. Note that the norm of $\mathfrak{X}$ is invariant under the scaling (1.9), that is,

$$
\left\|\left(\mathbf{u}_{\lambda}, v_{\lambda}, w_{\lambda}\right)\right\|_{\mathcal{X} \times\left[y_{a}\right]^{2}}=\|(\mathbf{u}, v, w)\|_{\chi \times\left[y_{a}\right]^{2}}
$$

Hence, by the uniqueness result of Theorem 1.3, the solution $(\mathbf{u}, v, w)$ of $(1.1)-(1.6)$ is selfsimilar. 


\section{Stability of Solutions}

In this short section, we prove Theorem 1.6. Let us pick up any two solutions $(\mathbf{u}, v, w)$ and $(\widetilde{\mathbf{u}}, \widetilde{v}, \widetilde{w})$ associated with initial conditions $\left(\mathbf{u}_{0}, v_{0}, w_{0}\right)$ and $\left(\widetilde{\mathbf{u}}_{0}, \widetilde{v}_{0}, \widetilde{w}_{0}\right)$, respectively, lying in $\left[P \mathcal{M}^{2}\right]^{3} \times\left[P M^{1}\right]^{2}$. As in the proof of Lemma 2.1, one has

$$
\begin{aligned}
& \left\|\int_{0}^{t} e^{(t-\tau) \Delta}\left(G_{1}(\mathbf{u}(\tau), v(\tau), w(\tau))-G_{1}(\tilde{\mathbf{u}}(\tau), \tilde{v}(\tau), \tilde{w}(\tau))\right) d \tau\right\|_{X} \\
& \quad \leq \eta\left[\|\mathbf{u}-\tilde{\mathbf{u}}\|_{\mathcal{X}}\left(\|\mathbf{u}\|_{\mathcal{X}}+\|\tilde{\mathbf{u}}\|_{\mathcal{X}}\right)+\|(v-\tilde{v}, w-\tilde{w})\|_{y_{a}}\left(\|(v, w)\|_{y_{a}}+\|(\tilde{v}, \tilde{w})\|_{y_{a}}\right)\right] \\
& \quad \leq 2 \eta M^{2},
\end{aligned}
$$

where $M=\max \left\{\|(\mathbf{u}, v, w)\|_{\mathfrak{X}},\|(\tilde{\mathbf{u}}, \tilde{v}, \widetilde{w})\|_{\mathfrak{X}},\|(\mathbf{u}-\tilde{\mathbf{u}}, v-\widetilde{v}, w-\widetilde{w})\|_{\mathfrak{X}}\right\}$. Similarly,

$$
\begin{aligned}
& \left\|\int_{0}^{t} e^{(t-\tau) \Delta}\left(G_{2}(\mathbf{u}(\tau), v(\tau), w(\tau))-G_{2}(\tilde{\mathbf{u}}(\tau), \tilde{v}(\tau), \tilde{w}(\tau))\right) d \tau\right\|_{y_{a}} \leq 2 \eta M^{2}, \\
& \left\|\int_{0}^{t} e^{(t-\tau) \Delta}\left(G_{3}(\mathbf{u}(\tau), v(\tau), w(\tau))-G_{3}(\tilde{\mathbf{u}}(\tau), \tilde{v}(\tau), \tilde{w}(\tau))\right) d \tau\right\|_{y_{a}} \leq 2 \eta M^{2} .
\end{aligned}
$$

Now, we subtract the integral equation (1.14) for $(\tilde{\mathbf{u}}, \tilde{v}, \tilde{w})$ from the analogous expression for $(\mathbf{u}, v, w)$, using the definition of the norm of $\mathfrak{X}$ and (3.1)-(3.2), we obtain

$$
\begin{aligned}
\|(\mathbf{u}-\tilde{\mathbf{u}}, v-\tilde{v}, w-\tilde{w})\|_{X} & \leq\left\|e^{t \Delta}\left(\mathbf{u}_{0}-\widetilde{\mathbf{u}}_{0}, v_{0}-\widetilde{v}_{0}, w_{0}-\widetilde{w}_{0}\right)\right\|_{\mathfrak{X}}+2 \eta M^{2} \\
& \leq \eta\left\|\left(\mathbf{u}_{0}-\widetilde{\mathbf{u}}_{0}, v_{0}-\widetilde{v}_{0}, w_{0}-\widetilde{w}_{0}\right)\right\|_{\left[p M^{2}\right]^{3} \times\left[p M^{1}\right]^{2}}+2 \eta M^{2} .
\end{aligned}
$$

Applying the definition of $M$, (3.3) yields that

$$
M \leq \eta\left\|\left(\mathbf{u}_{0}-\tilde{\mathbf{u}}_{0}, v_{0}-\tilde{v}_{0}, w_{0}-\widetilde{w}_{0}\right)\right\|_{\left[p \mathcal{M}^{2}\right]^{3} \times\left[p \mathcal{M}^{1}\right]^{2}}+2 \eta M^{2}
$$

Since we have assumed that $\left\|\left(\mathbf{u}_{0}-\widetilde{\mathbf{u}}_{0}, v_{0}-\widetilde{v}_{0}, w_{0}-\widetilde{w}_{0}\right)\right\|_{\left[p M^{2}\right]^{3} \times\left[p M^{1}\right]^{2}}<1 /\left(8 \eta^{2}\right)$, the continuity argument implies that $M \leq M_{1}$, where $M_{1}$ is the smallest root corresponding to the following quadratic equation:

$$
2 \eta M^{2}-M+\eta\left\|\left(\mathbf{u}_{0}-\tilde{\mathbf{u}}_{0}, v_{0}-\widetilde{v}_{0}, w_{0}-\tilde{w}_{0}\right)\right\|_{\left[p \mathcal{M}^{2}\right]^{3} \times\left[p \mathcal{M}^{1}\right]^{2}}=0 .
$$

From (3.4) we know that this root satisfies

$$
M_{1} \leq 2 \eta\left\|\left(\mathbf{u}_{0}-\tilde{\mathbf{u}}_{0}, v_{0}-\widetilde{v}_{0}, w_{0}-\tilde{w}_{0}\right)\right\|_{\left[p \mathcal{M}^{2}\right]^{3} \times\left[p \mathcal{M}^{1}\right]^{2}}
$$

Since $M \leq M_{1}$, this last inequality yields that

$$
\|(\mathbf{u}-\tilde{\mathbf{u}}, v-\widetilde{v}, w-\widetilde{w})\|_{\mathfrak{X}} \leq 2 \eta\left\|\left(\mathbf{u}_{0}-\widetilde{\mathbf{u}}_{0}, v_{0}-\widetilde{v}_{0}, w_{0}-\widetilde{w}_{0}\right)\right\|_{\left[p \mathcal{M}^{2}\right]^{3} \times\left[p \mathcal{M}^{1}\right]^{2}}
$$

The proof of Theorem 1.6 is complete. 


\section{Large Time Behavior of Solutions}

We are now in a position to show Theorem 1.9 on the large time behavior of solutions to the system $(1.1)-(1.6)$. Let $(\mathbf{u}, v, w)$ and $(\tilde{\mathbf{u}}, \widetilde{v}, \widetilde{w})$ be two solutions of (1.1)-(1.6) constructed in Theorem 1.3 which correspond to initial conditions $\left(\mathbf{u}_{0}, v_{0}, w_{0}\right)$ and $\left(\widetilde{\mathbf{u}}_{0}, \widetilde{v}_{0}, \widetilde{w}_{0}\right)$, respectively. Let us recall that, by Lemma 2.1 , there exists a constant $2 \eta \varepsilon$, the radius of $\mathfrak{B}$, such that

$$
\|(\mathbf{u}, v, w)\|_{\mathfrak{X}} \leq 2 \eta \varepsilon, \quad\|(\widetilde{\mathbf{u}}, \widetilde{v}, \widetilde{w})\|_{\mathfrak{X}} \leq 2 \eta \varepsilon
$$

Now, to simplify the notations, we introduce the following two auxiliary functions:

$$
\begin{aligned}
h(t)= & \left\|e^{t \Delta}\left(\mathbf{u}_{0}-\widetilde{\mathbf{u}}_{0}, v_{0}-\widetilde{v}_{0}, w_{0}-\widetilde{w}_{0}\right)\right\|_{\left[p \mathcal{M}^{2}\right]^{3} \times\left[p \mathcal{M}^{1}\right]^{2}} \\
& +t^{(a-1) / 2}\left\|e^{t \Delta}\left(v_{0}-\widetilde{v}_{0}, w_{0}-\widetilde{w}_{0}\right)\right\|_{\left[p \mathcal{M}^{a}\right]^{2^{\prime}}} \\
l(t)= & \|(\mathbf{u}-\widetilde{\mathbf{u}}, v-\widetilde{v}, w-\widetilde{w})\|_{\left[p \mathcal{M}^{2}\right]^{3} \times\left[p \mathcal{M}^{1}\right]^{2}} \\
& +t^{(a-1) / 2}\|(v-\widetilde{v}, w-\widetilde{w})\|_{\left[p \mathcal{M}^{a}\right]^{2} .}
\end{aligned}
$$

We first assume that (1.26) holds. It follows immediately from Lemma 2.1 that

$$
h(t) \in L^{\infty}([0, \infty)), \quad \lim _{t \rightarrow \infty} h(t)=0 .
$$

By calculating the norm $\|\cdot\|_{\left[p \mathscr{N}^{2}\right]^{3}}$ of $\mathbf{u}-\tilde{\mathbf{u}}$, we can easily get

$$
\begin{aligned}
& \|\mathbf{u}-\tilde{\mathbf{u}}\|_{\left[p \mathcal{M}^{2}\right]^{3}} \leq\left\|e^{t \Delta}\left(\mathbf{u}_{0}-\widetilde{\mathbf{u}}_{0}\right)\right\|_{\left[p \mathcal{M}^{2}\right]^{3}}+\left\|\int_{0}^{t} e^{(t-\tau) \Delta} \mathbb{P} \nabla(\mathbf{u} \otimes \mathbf{u}-\tilde{\mathbf{u}} \otimes \tilde{\mathbf{u}}) d \tau\right\|_{\left[p \mathcal{M}^{2}\right]^{3}} \\
& +\| \int_{0}^{t} e^{(t-\tau) \Delta} \\
& \times \mathbb{P}\left[(v-w)\left(\nabla(-\Delta)^{-1}(w-v)\right)-(\tilde{v}-\tilde{w})\left(\nabla(-\Delta)^{-1}(\tilde{w}-\tilde{v})\right)\right] d \tau \|_{\left[p M^{2}\right]^{3}} \\
& =I_{0}+I_{1}+I_{2} .
\end{aligned}
$$

For $I_{1}$, let $\delta \in(0,1)$ be a constant to be chosen later, we decompose the integral $\int_{0}^{t} \cdots d \tau$ into $\int_{0}^{\delta t} \cdots d \tau+\int_{\delta t}^{t} \cdots d \tau$ and estimate each term separately:

$$
I_{1} \lesssim \sup _{\xi \in \mathbb{R}^{3}}\left[\int_{0}^{\delta t}+\int_{\delta t}^{t}\right]|\xi|^{2} e^{-(t-\tau)|\xi|^{2}}\left(\|\mathbf{u}\|_{\left[p \mathcal{M}^{2}\right]^{3}}+\|\tilde{\mathbf{u}}\|_{\left[p \mathcal{M}^{2}\right]^{3}}\right)\|\mathbf{u}-\tilde{\mathbf{u}}\|_{\left[p \mathcal{M}^{2}\right]^{3}} d \tau=I_{11}+I_{12}
$$


For $I_{11}$, we change the variables $\tau=t s$ and use the following identity:

$$
\sup _{\xi \in \mathbb{R}^{3}}|\xi|^{2} e^{-(1-s) t|\xi|^{2}}=\frac{1}{(1-s) t} \cdot \sup _{\sigma \in \mathbb{R}^{3}}|\sigma|^{2} e^{-|\sigma|^{2}}=\frac{1}{(1-s) t e}
$$

Thus, from (4.1), one has

$$
I_{11} \lesssim 4 \eta \varepsilon \int_{0}^{\delta}(1-s)^{-1}\|\mathbf{u}(t s)-\tilde{\mathbf{u}}(t s)\|_{\left[p \mathscr{N}^{2}\right]^{3}} d s
$$

For $I_{12}$, we can estimate it directly that

$$
I_{12} \lesssim 4 \eta \varepsilon \sup _{\delta t \leq \tau \leq t}\|\mathbf{u}(\tau)-\widetilde{\mathbf{u}}(\tau)\|_{\left[p \mathscr{M}^{2}\right]^{3}}
$$

Hence, it follows immediately from (4.7) and (4.8) that

$$
I_{1} \lesssim 4 \eta \varepsilon\left(\int_{0}^{\delta}(1-s)^{-1}\|\mathbf{u}(t s)-\widetilde{\mathbf{u}}(t s)\|_{\left[p \mathscr{M}^{2}\right]^{3}} d s+\sup _{\delta t \leq \tau \leq t}\|\mathbf{u}(\tau)-\widetilde{\mathbf{u}}(\tau)\|_{\left[p \mathscr{M}^{2}\right]^{3}}\right)
$$

Now, we compute $I_{2}$, by using the same argument, we obtain

$$
\begin{aligned}
I_{2} \lesssim & \sup _{\xi \in \mathbb{R}^{3}}\left[\int_{0}^{\delta t}+\int_{\delta t}^{t}\right]|\xi|^{4-2 a} e^{-(t-\tau)|\xi|^{2}} \tau^{-(a-1)} \\
& \times\left(\tau^{(a-1) / 2}\|(v, w)\|_{\left[p \mathcal{M}^{a}\right]^{2}}+\tau^{(a-1) / 2}\|(\widetilde{v}, \widetilde{w})\|_{\left[p \mathscr{M}^{a}\right]^{2}}\right) \\
& \times\left(\tau^{(a-1) / 2}\|(v-\widetilde{v}, w-\widetilde{w})\|_{\left[p \mathcal{M}^{a}\right]^{2}}\right) d \tau \\
= & I_{21}+I_{22} .
\end{aligned}
$$

For $I_{21}$, we change the variables $\tau=t s$, and from (4.1),

$$
I_{21} \lesssim 4 \eta \mathcal{E} \int_{0}^{\delta}(1-s)^{-(2-a)} s^{-(a-1)}\left((t s)^{(a-1) / 2}\|(v(t s)-\widetilde{v}(t s), w(t s)-\widetilde{w}(t s))\|_{\left[p \Re^{a}\right]^{2}}\right) d s
$$

For $I_{22}$, we estimate it directly that

$$
I_{22} \lesssim 4 \eta \varepsilon \sup _{\delta t \leq \tau \leq t}\|(v(\tau)-\widetilde{v}(\tau), w(\tau)-\tilde{w}(\tau))\|_{\left[p \mathcal{M}^{a}\right]^{2}}
$$


From (4.11) and (4.12), we get

$$
\begin{aligned}
& I_{2} \lesssim 4 \eta \varepsilon \\
& \quad\left(\int_{0}^{\delta}(1-s)^{-(2-a)} s^{-(a-1)}\left((t s)^{(a-1) / 2}\|(v(t s)-\widetilde{v}(t s), w(t s)-\widetilde{w}(t s))\|_{\left[p M^{a}\right]^{2}}\right) d s\right. \\
& \left.\quad+\sup _{\delta t \leq \tau \leq t}\|(v(\tau)-\widetilde{v}(\tau), w(\tau)-\widetilde{w}(\tau))\|_{\left[p \mathcal{M}^{a}\right]^{2}}\right) .
\end{aligned}
$$

Combining the above estimates (4.9) and (4.13), we have already proved that

$$
\begin{aligned}
& \|\mathbf{u}-\tilde{\mathbf{u}}\|_{\left[p \mathscr{M}^{2}\right]^{3}} \lesssim\left\|e^{t \Delta}\left(\mathbf{u}_{0}-\tilde{\mathbf{u}}_{0}\right)\right\|_{\left[p \mathscr{N}^{2}\right]^{3}}+4 \eta \varepsilon \\
& \times\left[\int_{0}^{\delta}(1-s)^{-1}\|\mathbf{u}(t s)-\tilde{\mathbf{u}}(t s)\|_{\left[p \mathscr{M}^{2}\right]^{3}} d s+\sup _{\delta t \leq \tau \leq t}\|\mathbf{u}(\tau)-\tilde{\mathbf{u}}(\tau)\|_{\left[P \mathscr{M}^{2}\right]^{3}}\right. \\
& +\int_{0}^{\mathcal{\delta}}(1-s)^{-(2-a)} s^{-(a-1)}\left((t s)^{(a-1) / 2}\|(v(t s)-\widetilde{v}(t s), w(t s)-\tilde{w}(t s))\|_{\left[p M^{a}\right]^{2}}\right) d s \\
& \left.+\sup _{\delta t \leq \tau \leq t}\|(v(\tau)-\widetilde{v}(\tau), w(\tau)-\widetilde{w}(\tau))\|_{\left[p \mathcal{M}^{a}\right]^{2}}\right] .
\end{aligned}
$$

By using the analogous argument above, we can estimate $v-\widetilde{v}$ and obtain the following estimate:

$$
\begin{aligned}
\|v-\tilde{v}\|_{p \mathcal{M}^{1}}+t^{(a-1) / 2}\|v-\tilde{v}\|_{p \mathcal{M}^{a}} \lesssim & \left\|e^{t \Delta}\left(v_{0}-\widetilde{v}_{0}\right)\right\|_{p \mathcal{M}^{1}}+t^{(a-1) / 2}\left\|e^{t \Delta}\left(v_{0}-\tilde{v}_{0}\right)\right\|_{p \mathcal{M}^{a}}+4 \eta \varepsilon \\
& \times\left[\int_{0}^{\delta}(1-s)^{-(3-a) / 2} s^{-(a-1) / 2}\right. \\
& \times\left(\|\mathbf{u}(t s)-\tilde{\mathbf{u}}(t s)\|_{\left[p \mathcal{M}^{2}\right]^{3}}\right. \\
& \left.+(t s)^{(a-1) / 2}\|v(t s)-\widetilde{v}(t s)\|_{p \mathcal{M}^{a}}\right) d s \\
& +\int_{0}^{\delta}(1-s)^{-(2-a)} s^{-(a-1)} \\
& \times\left((t s)^{(a-1) / 2}\|(v(t s)-\tilde{v}(t s), w(t s)-\tilde{w}(t s))\|_{\left[p \mathcal{M}^{a}\right]^{2}}\right) d s \\
& +\int_{0}^{\delta}(1-s)^{-1} s^{-(a-1) / 2} \\
& \times\left(\|\mathbf{u}(t s)-\widetilde{\mathbf{u}}(t s)\|_{\left[D \mathcal{M}^{2}\right]^{3}}\right.
\end{aligned}
$$




$$
\begin{aligned}
& \left.\quad+(t s)^{(a-1) / 2}\|v(t s)-\tilde{v}(t s)\|_{p \mathcal{M}^{a}}\right) d s \\
& +\int_{0}^{\delta}(1-s)^{-(3-a) / 2} s^{-(a-1)} \\
& \times\left((t s)^{(a-1) / 2}\|(v(t s)-\tilde{v}(t s), w(t s)-\tilde{w}(t s))\|_{\left[p \mathcal{M}^{a}\right]^{2}}\right) d s \\
& +\sup _{\delta t \leq \tau \leq t}\left(\|\mathbf{u}(\tau)-\tilde{\mathbf{u}}(\tau)\|_{\left[p \mathcal{M}^{2}\right]^{3}}\right. \\
& \left.\quad+\tau^{(a-1) / 2} \|\left(v(\tau)-\tilde{v}(\tau), w(\tau)-\tilde{w}(\tau) \|_{\left[p M^{a}\right]^{2}}\right)\right] .
\end{aligned}
$$

The estimate of $w-\widetilde{w}$ has exactly the same form as (4.15). Now, let

$$
M=\limsup _{t \rightarrow \infty} l(t)=\lim _{k \in \mathbb{N}, k \rightarrow \infty} \sup _{t \geq k} l(t) .
$$

In order to prove (1.27), it suffices to prove $M=0$. Note that from (4.1) we know that $M$ is nonnegative and finite. Hence, by applying the Lebesgue dominated convergence theorem to (4.14), (4.15), and the same estimate as (4.15) for $w-\tilde{w}$ and using the assumption (1.26) we obtain

$$
M \lesssim 4 \eta \varepsilon(F(\delta)+1) M
$$

where $F(\delta)$ is defined by

$$
\begin{aligned}
F(\delta)= & \log \left(\frac{1}{(1-\delta)}\right)+\int_{0}^{\delta}(1-s)^{-(2-a)} s^{-(a-1)} d s \\
& +\int_{0}^{\delta}(1-s)^{-(3-a) / 2} s^{-(a-1) / 2} d s \\
& +\int_{0}^{\delta}(1-s)^{-1} s^{-(a-1) / 2} d s+\int_{0}^{\delta}(1-s)^{-(3-a) / 2} s^{-(a-1)} d s .
\end{aligned}
$$

Thus, there exists a universal constant $\tilde{\eta}$ which may depend on $\eta$ such that

$$
M \leq \tilde{\eta} \varepsilon(F(\delta)+1) M
$$

Since we have assumed that $\varepsilon \tilde{\eta}<1$, we can choose $\delta$ sufficiently small such that $\tilde{\eta} \varepsilon(F(\delta)+1)<$ 1 by the fact that $\lim _{\delta \rightarrow 0} F(\delta)=0$. This implies $M=0$ by (4.19). We complete the proof of (1.27). 
Conversely, we assume that (1.27) holds. Note that from (4.1) one has

$$
l(t) \in L^{\infty}([0, \infty)), \quad \lim _{t \rightarrow \infty} l(t)=0
$$

We need to prove (1.26). Repeat calculations similar to the proofs of (4.14) and (4.15), and, from the boundedness of $(\mathbf{u}, v, w)$ and $(\widetilde{\mathbf{u}}, \widetilde{v}, \widetilde{w})$ in $(4.1)$, we can obtain the following estimate:

$$
h(t) \leq \tilde{\eta} \varepsilon(F(\delta)+1) l(t)
$$

It is obvious that $\tilde{\eta} \varepsilon(F(\delta)+1)$ is bounded and independent of $t$, so (1.26) follows immediately from (1.27) and (4.21). This proves Theorem 1.9.

\section{Acknowledgments}

This work is supported by the China National Natural Science Foundation under the Grant no. 11171357. The authors would like to thank the anonymous referee for invaluable suggestions.

\section{References}

[1] I. Rubinstein, Electro-Diffusion of Ions, vol. 11 of SIAM Studies in Applied Mathematics, Society for Industrial and Applied Mathematics, Philadelphia, Pa, USA, 1990.

[2] J. Leray, "Sur le mouvement d'un liquide visqueux emplissant l'espace," Acta Mathematica, vol. 63, no. 1, pp. 193-248, 1934.

[3] J. Bourgain and N. Pavlović, "Ill-posedness of the Navier-Stokes equations in a critical space in 3D," Journal of Functional Analysis, vol. 255, no. 9, pp. 2233-2247, 2008.

[4] M. Cannone, "Harmonic analysis tools for solving the incompressible Navier-Stokes equations," in Handbook of Mathematical Fluid Dynamics, S. Friedlander and D. Serre, Eds., vol. 3, pp. 161-244, Elsevier, Amsterdam, The Netherlands, 2004.

[5] H. Fujita and T. Kato, "On the Navier-Stokes initial value problem. I," Archive for Rational Mechanics and Analysis, vol. 16, pp. 269-315, 1964.

[6] T. Kato, "Strong $L^{p}$-solutions of the Navier-Stokes equation in $\mathbb{R}^{m}$, with applications to weak solutions," Mathematische Zeitschrift, vol. 187, no. 4, pp. 471-480, 1984.

[7] H. Koch and D. Tataru, "Well-posedness for the Navier-Stokes equations," Advances in Mathematics, vol. 157, no. 1, pp. 22-35, 2001.

[8] P. G. Lemarie-Rieusset, Recent Developments in the Navier-Stokes Problem, vol. 431 of Chapman E Hall/ CRC Research Notes in Mathematics, Chapman \& Hall/CRC, Boca Raton, Fla, USA, 2002.

[9] P. Debye and E. Hückel, "Zur theorie der elektrolyte, II: das grenzgesetz für die elektrische leitfähigkeit," Physik Zeitschrift, vol. 24, pp. 305-325, 1923.

[10] P. Biler, W. Hebisch, and T. Nadzieja, "The Debye system: existence and large time behavior of solutions," Nonlinear Analysis, vol. 23, no. 9, pp. 1189-1209, 1994.

[11] P. Biler and J. Dolbeault, "Long time behavior of solutions of Nernst-Planck and Debye-Hückel driftdiffusion systems," Annales Henri Poincaré, vol. 1, no. 3, pp. 461-472, 2000.

[12] H. Gajewski and K. Gröger, "On the basic equations for carrier transport in semiconductors," Journal of Mathematical Analysis and Applications, vol. 113, no. 1, pp. 12-35, 1986.

[13] G. Karch, "Scaling in nonlinear parabolic equations," Journal of Mathematical Analysis and Applications, vol. 234, no. 2, pp. 534-558, 1999.

[14] M. Kurokiba and T. Ogawa, "Well-posedness for the drift-diffusion system in $L^{p}$ arising from the semiconductor device simulation," Journal of Mathematical Analysis and Applications, vol. 342, no. 2, pp. 1052-1067, 2008. 
[15] T. Ogawa and S. Shimizu, "The drift-diffusion system in two-dimensional critical Hardy space," Journal of Functional Analysis, vol. 255, no. 5, pp. 1107-1138, 2008.

[16] T. Ogawa and M. Yamamoto, "Asymptotic behavior of solutions to drift-diffusion system with generalized dissipation," Mathematical Models E Methods in Applied Sciences, vol. 19, no. 6, pp. 939 967, 2009.

[17] J. W. Jerome, "Analytical approaches to charge transport in a moving medium," Transport Theory and Statistical Physics, vol. 31, no. 4-6, pp. 333-366, 2002.

[18] M. Schmuck, "Analysis of the Navier-Stokes-Nernst-Planck-Poisson system," Mathematical Models $\mathcal{E}$ Methods in Applied Sciences, vol. 19, no. 6, pp. 993-1015, 2009.

[19] R. J. Ryham, "Existence, uniqueness, regularity and long-term behavior for dissipative systems modeling electrohydrodynamics," submitted to Analysis of PDEs, http://arxiv.org/abs/0910.4973v1.

[20] J. Zhao, C. Deng, and S. Cui, "Well-posedness of a dissipative system modeling electrohydrodynamics in Lebesgue spaces," Differential Equations E Applications, vol. 3, no. 3, pp. 427-448, 2011.

[21] M. Longaretti, B. Chini, J. W. Jerome, and R. Sacco, "Electrochemical modeling and characterization of voltage operated channels in nano-bio-electronics," Sensor Letters, vol. 6, no. 1, pp. 49-56, 2008.

[22] M. Longaretti, B. Chini, J. W. Jerome, and R. Sacco, "Computational modeling and simulation of complex systems in bio-electronics," Journal of Computational Electronics, vol. 7, no. 1, pp. 10-13, 2008.

[23] M. Longaretti, G. Marino, B. Chini, J. W. Jerome, and R. Sacco, "Computational models in nanobioelectronics: simulation of ionic transport in voltage operated channels," Journal of Nanoscience and Nanotechnology, vol. 8, no. 7, pp. 3686-3694, 2008.

[24] G. Tian and Z. Xin, "One-point singular solutions to the Navier-Stokes equations," Topological Methods in Nonlinear Analysis, vol. 11, no. 1, pp. 135-145, 1998.

[25] P. Biler, M. Cannone, I. A. Guerra, and G. Karch, “Global regular and singular solutions for a model of gravitating particles," Mathematische Annalen, vol. 330, no. 4, pp. 693-708, 2004.

[26] G. Benfatto, R. Esposito, and M. Pulvirenti, "Planar Navier-Stokes flow for singular initial data," Nonlinear Analysis, vol. 9, no. 6, pp. 533-545, 1985.

[27] M. Cannone and G. Karch, "Smooth or singular solutions to the Navier-Stokes system?" Journal of Differential Equations, vol. 197, no. 2, pp. 247-274, 2004.

[28] Y. Le Jan and A. S. Sznitman, "Stochastic cascades and 3-dimensional Navier-Stokes equations," Probability Theory and Related Fields, vol. 109, no. 3, pp. 343-366, 1997.

[29] E. M. Stein, Singular Integrals and Differentiability Properties of Functions, Princeton Mathematical Series, Princeton University Press, Princeton, NJ, USA, 1970.

[30] M. Yamazaki, "The Navier-Stokes equations in the weak- $L^{n}$ space with time-dependent external force," Mathematische Annalen, vol. 317, no. 4, pp. 635-675, 2000. 


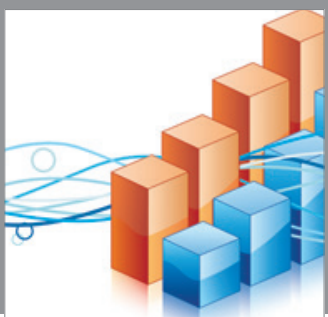

Advances in

Operations Research

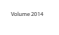

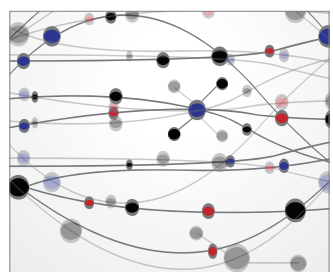

\section{The Scientific} World Journal
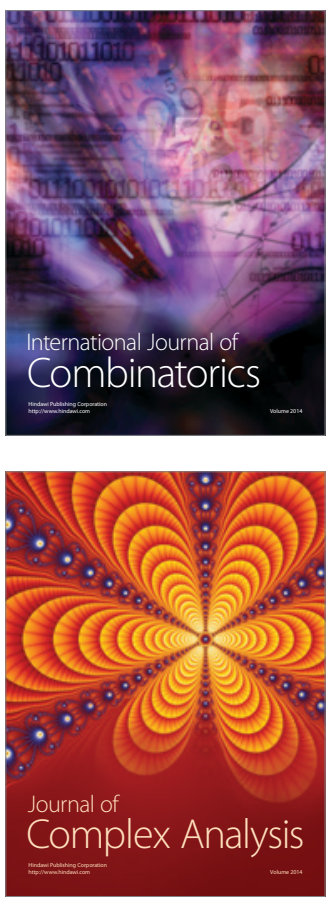

International Journal of

Mathematics and

Mathematical

Sciences
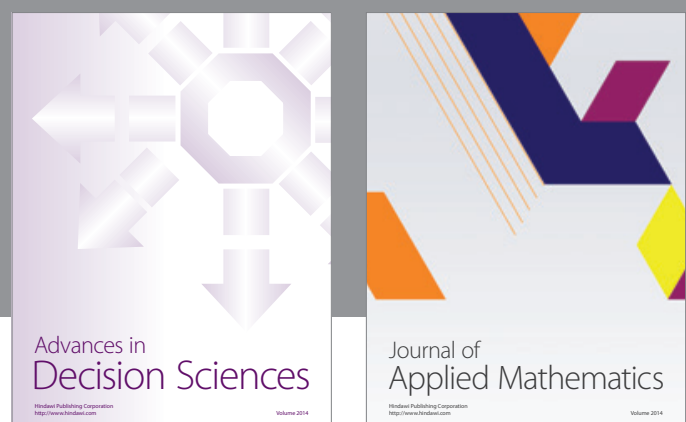

Journal of

Applied Mathematics
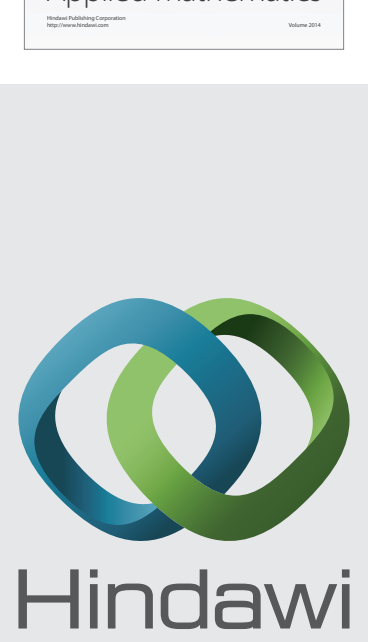

Submit your manuscripts at http://www.hindawi.com
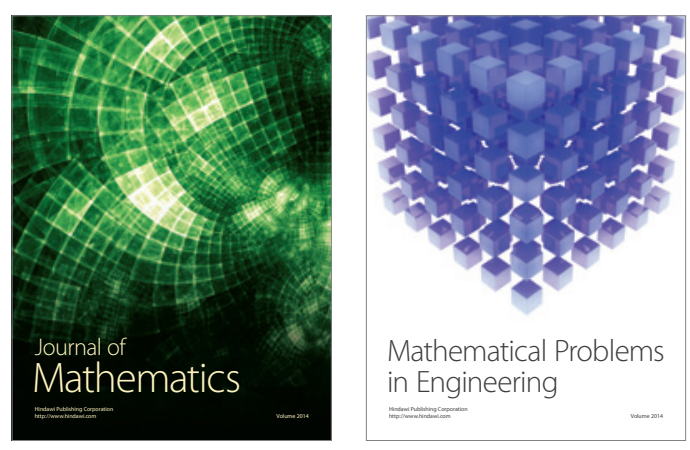

Mathematical Problems in Engineering
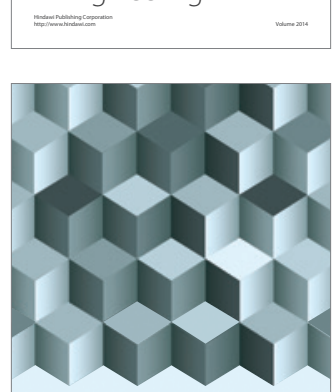

Journal of

Function Spaces
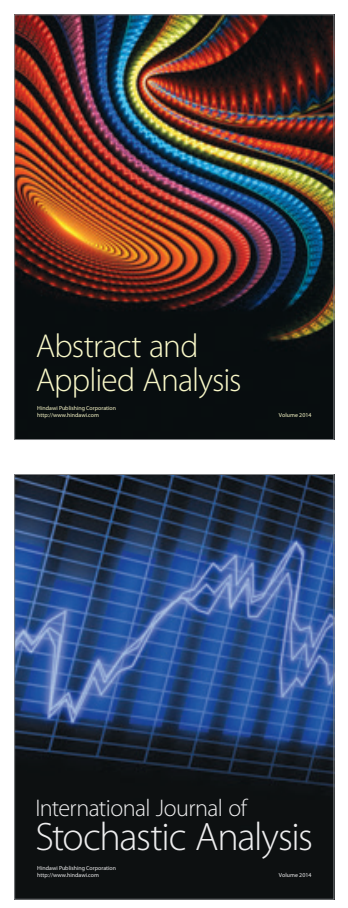

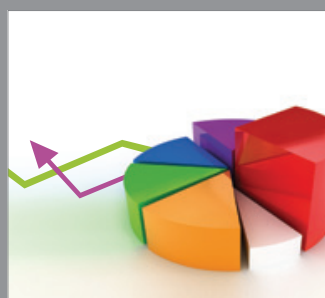

ournal of

Probability and Statistics

Promensencen
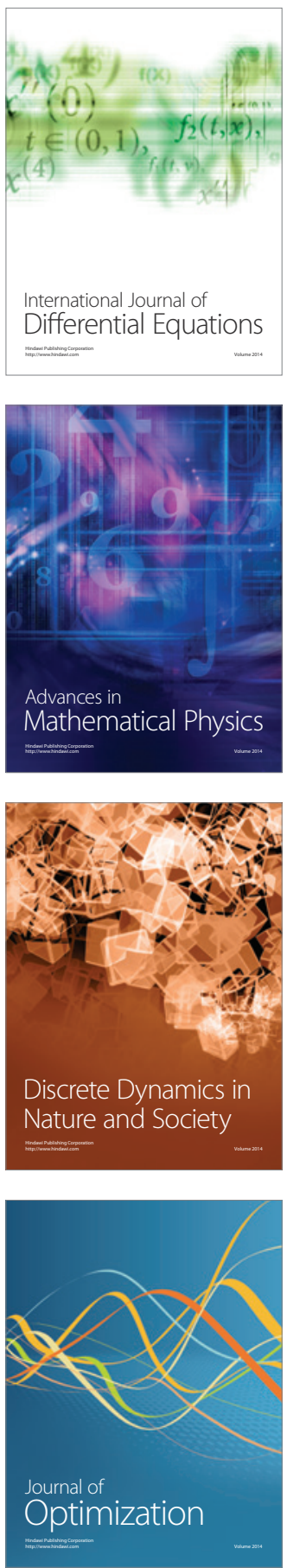